\title{
Influência dos Parâmetros do Ciclo de Prensagem na Qualidade do Revestimento de Painéis Compensados Plastificados
}

\author{
Felipe Gustavo Sanches ${ }^{1}$, Setsuo Iwakiri ${ }^{1}$ \\ ${ }^{1}$ Departamento de Engenharia e Tecnologia Florestal, Universidade Federal do Paraná - UFPR, Curitiba/PR, Brasil
}

\begin{abstract}
RESUMO
O objetivo deste trabalho foi avaliar a influência dos parâmetros do processo de revestimento na qualidade de painéis compensados plastificados, através das variáveis: gramatura de filme fenólico, temperatura de prensagem, pressão específica e tempo de prensagem. A qualidade de revestimento foi avaliada através de 48 painéis submetidos a ensaios de resistência a abrasão e absorção de vapor, seguidos por interpretação dos resultados por análise estatística fatorial. Melhores resultados de abrasão foram obtidos para filme fenólico de maior gramatura. Os parâmetros de prensagem não afetaram a absorção de vapor. Recomenda-se realizar o revestimento fenólico nas seguintes condições: gramatura: $215 \mathrm{~g} / \mathrm{m}^{2}$; temperatura: $130{ }^{\circ} \mathrm{C}$; pressão específica: $16 \mathrm{kgf} / \mathrm{cm}^{2}$ : tempo de prensagem: 6 minutos.
\end{abstract}

Palavras-chave: filme fenólico, fôrmas de concreto, processo de prensagem.

\section{Coating Quality of Film-faced Plywood as a Function of Pressing Cycle}

\begin{abstract}
The aim of this study was to evaluate the effect of process parameters on the coating quality of film-faced plywood using variables such as phenolic film grammage, press temperature, specific pressure, and pressing time. Coating quality was evaluated in 48 panels tested by resistance to abrasion and steam absorption, followed by statistical factorial analysis. The highest phenolic film grammage showed better results in the abrasion test. Pressing parameters did not affect the absorption of steam. We recommend the performance of phenolic coating under the following conditions: grammage: $215 \mathrm{~g} / \mathrm{m}^{2}$, temperature: $130{ }^{\circ} \mathrm{C}$; specific pressure: $16 \mathrm{kgf} / \mathrm{cm}^{2}$ : pressing time: 6 minutes.
\end{abstract}

Keywords: phenolic film, concrete formworks, pressing process. 


\section{INTRODUÇÃO}

Deacordocoma Associação BrasileiradaIndústria de Madeira Processada Mecanicamente - ABIMCI (2011), há várias décadas as indústrias de painéis compensados já destinavam parte de sua produção para fabricação de compensado plastificado, visando a sua utilização como formas de concreto na construção civil.

Após passar por períodos de alta na produção e no consumo, o setor de indústrias de compensados foi atingido pela conjuntura econômica, com a desvalorização do real a partir de 2004, e pela crise internacional de 2008, resultando em fechamento de muitas unidades fabris. Entretanto, algumas indústrias buscaram alternativas aos compensados convencionais, como os painéis plastificados, com maior valor agregado (Polzl, 2011).

De acordo com Costa Junior (2008), o compensado plastificado é um painel compensado multilaminado, com suas faces revestidas com filme fenólico. É bastante utilizado na execução de fôrmas para concreto aparente, em função do alto desempenho dos painéis na obtenção de superfícies mais regulares e menos ásperas, com elevado índice de reaproveitamento. Segundo Araujo (2010), o compensado plastificado é recomendado para uso externo e fôrmas para concreto, sendo economicamente viável por possuir um elevado índice de reutilizações.

O filme fenólico ou tegofilme é obtido através da impregnação de um papel Kraft com resina fenol-formaldeído com gramatura na faixa de 120 a $220 \mathrm{~g} / \mathrm{m}^{2}$ (Iwakiri et al., 2004). A impregnação do papel com esse tipo de resina proporciona excelente resistência aos solventes, ácidos e sais, além de melhorar as propriedades de isolamento elétrico, em razão da baixa capacidade de absorção de umidade (Silva, 2008)

O processo de 'placagem' consiste em recobrir a face do compensado tradicional com filme fenólico e realizar a prensagem numa prensa quente para fusão das duas superfícies. Iwakiri et al. (2004) recomendam que o compensado seja prensado com pressão específica de $15 \mathrm{kgf} / \mathrm{cm}^{2}$, temperatura a partir de $140{ }^{\circ} \mathrm{C}$ e tempo de prensagem na faixa de 4 a 8 minutos.
De acordo com a Coveright Surfaces (2011), os filmes são normalmente prensados em um painel compensado acabado. $\mathrm{O}$ fabricante recomenda o uso de pratos de aço inoxidável, porém aço cromado e alumínio também podem ser utilizados.

O compensado plastificado ganhou mercado dentro do setor de transportes, sendo utilizado como assoalhos para caminhões e ônibus. Destaca-se também o seu uso na construção civil, em função da qualidade de acabamento das fôrmas de concreto e da resistência às condições de alta umidade, além da maior reutilização, a qual, consequentemente, reduz os custos das obras (Stamato, 1998).

Tendo em vista a carência de publicações científicas abordando esse tema, este trabalho foi desenvolvido com o objetivo de avaliar os efeitos da gramatura do filme fenólico, da temperatura, da pressão específica e do tempo de prensagem na qualidade do compensado plastificado.

\section{MATERIAL E MÉTODOS}

Foram utilizados compensados fenólicos de Pinus spp. com $18 \mathrm{~mm}$ de espessura, compostos de nove lâminas. Seis painéis foram retirados aleatoriamente da produção da empresa Formaplan - Formas Planejadas Ltda. Para revestimento, foram utilizados filmes fenólicos com gramaturas de 120,165 e $215 \mathrm{~g} / \mathrm{m}^{2}$, denominados comercialmente pelo fabricante como TPS 384, TPS 558 e TPS 758, respectivamente. Para prensagem do filme fenólico, foram utilizadas placas de aço inoxidável com dimensões de $60 \times 60 \times 2 \mathrm{~mm}$.

As variáveis de estudo foram: gramatura de 120,165 e $215 \mathrm{~g} / \mathrm{m}^{2}$, temperatura de prensagem de 130 e $140^{\circ} \mathrm{C}$, pressão específica de 16 e $18 \mathrm{kgf} / \mathrm{cm}^{2}$, e tempo de prensagem de 6 e 8 minutos, conforme delineamento experimental apresentado na Tabela 1.

De cada painel, foram retiradas oito placas com dimensões de $50 \times 50 \mathrm{~cm}$ para a aplicação do filme fenólico, perfazendo um total de 48 placas. As placas foram colocadas entre as chapas de aço e o filme fenólico nas posições inferior e superior, e prensadas numa prensa de laboratório. Após a prensagem, foram retirados os corpos de prova para a realização dos ensaios laboratoriais, sendo dois corpos de prova de $10 \times 10 \mathrm{~cm}$ para os ensaios de abrasão e um corpo 
Tabela 1. Delineamento experimental utilizado para avaliar a qualidade de revestimento de painéis compensados com filme fenólico.

Table 1. Experimental design used to evaluate the quality of plywood coating with phenolic film.

\begin{tabular}{|c|c|c|c|c|c|}
\hline Tratamento & $\begin{array}{l}\text { Gramatura } \\
\qquad\left(\mathrm{g} / \mathrm{m}^{2}\right)\end{array}$ & $\begin{array}{c}\text { Temperatura } \\
\left({ }^{\circ} \mathrm{C}\right)\end{array}$ & $\begin{array}{c}\text { Pressão específica } \\
\left(\mathbf{k g f} / \mathrm{cm}^{2}\right)\end{array}$ & $\begin{array}{l}\text { Tempo de prensagem } \\
\text { (min) }\end{array}$ & Repetições \\
\hline $\mathrm{T} 1$ & 120 & 130 & 16 & 6 & 2 \\
\hline $\mathrm{T} 2$ & 120 & 130 & 16 & 8 & 2 \\
\hline T3 & 120 & 130 & 18 & 6 & 2 \\
\hline $\mathrm{T} 4$ & 120 & 130 & 18 & 8 & 2 \\
\hline T5 & 120 & 140 & 16 & 6 & 2 \\
\hline T6 & 120 & 140 & 16 & 8 & 2 \\
\hline T7 & 120 & 140 & 18 & 6 & 2 \\
\hline $\mathrm{T} 8$ & 120 & 140 & 18 & 8 & 2 \\
\hline T9 & 165 & 130 & 16 & 6 & 2 \\
\hline $\mathrm{T} 10$ & 165 & 130 & 16 & 8 & 2 \\
\hline T11 & 165 & 130 & 18 & 6 & 2 \\
\hline T12 & 165 & 130 & 18 & 8 & 2 \\
\hline T13 & 165 & 140 & 16 & 6 & 2 \\
\hline T14 & 165 & 140 & 16 & 8 & 2 \\
\hline T15 & 165 & 140 & 18 & 6 & 2 \\
\hline T16 & 165 & 140 & 18 & 8 & 2 \\
\hline T17 & 215 & 130 & 16 & 6 & 2 \\
\hline T18 & 215 & 130 & 16 & 8 & 2 \\
\hline T19 & 215 & 130 & 18 & 6 & 2 \\
\hline $\mathrm{T} 20$ & 215 & 130 & 18 & 8 & 2 \\
\hline $\mathrm{T} 21$ & 215 & 140 & 16 & 6 & 2 \\
\hline $\mathrm{T} 22$ & 215 & 140 & 16 & 8 & 2 \\
\hline T23 & 215 & 140 & 18 & 6 & 2 \\
\hline \multirow[t]{2}{*}{$\mathrm{T} 24$} & 215 & 140 & 18 & 8 & 2 \\
\hline & & & & Total & 48 \\
\hline
\end{tabular}

de prova de $15 \times 15 \mathrm{~cm}$ para ensaios de absorção de vapor.

Nos ensaios de abrasão, as tiras abrasivas foram colocadas nas rodas de borracha do equipamento e fixadas por parafuso. $O$ número de ciclos foi determinado a partir da exposição de $50 \%$ da chapa de madeira. As tiras abrasivas foram trocadas a cada 500 ciclos completos. $\mathrm{O}$ aspirador de pó teve a função de retirar o pó gerado no processo abrasivo, além de evitar a impregnação de pós nas tiras abrasivas, o que as torna menos ásperas (Figura 1A-B).

No ensaio de absorção de vapor, foi verificado o acréscimo em massa resultante da passagem de vapor através do filme fenólico. Após a limpeza da superfície e a pesagem da amostra, a mesma foi colocada sobre o Erlemeyer de $500 \mathrm{~mL}$ contendo água previamente aquecida à temperatura de $100{ }^{\circ} \mathrm{C}$
(Figura 1C-D). As amostras foram retiradas e pesadas após 1 e 2 horas de exposição ao vapor, para a determinação da absorção de vapor (Equação 1).

$A V_{t}=\frac{P_{t}-P_{\text {inicial }}}{r^{2} \pi}$

em que: $A V \mathrm{~T}=$ absorção de vapor após $t$ horas de exposição ao vapor $\left(\mathrm{g} / \mathrm{m}^{2}\right) ; P \mathrm{~T}=$ peso da amostra após $t$ horas de exposição ao vapor (g); PINICIAL = peso inicial da amostra, ou seja, antes da exposição ao vapor $(\mathrm{g}) ; r=\operatorname{raio}(\mathrm{m})$.

Para a interpretação dos resultados, foi realizada a análise estatística fatorial. Inicialmente, foi utilizada a análise de variância com quatro fatores (gramatura, temperatura, pressão e tempo) para as variáveisresposta: resistência à abrasão e absorção de vapor em 1 e 2 horas. 

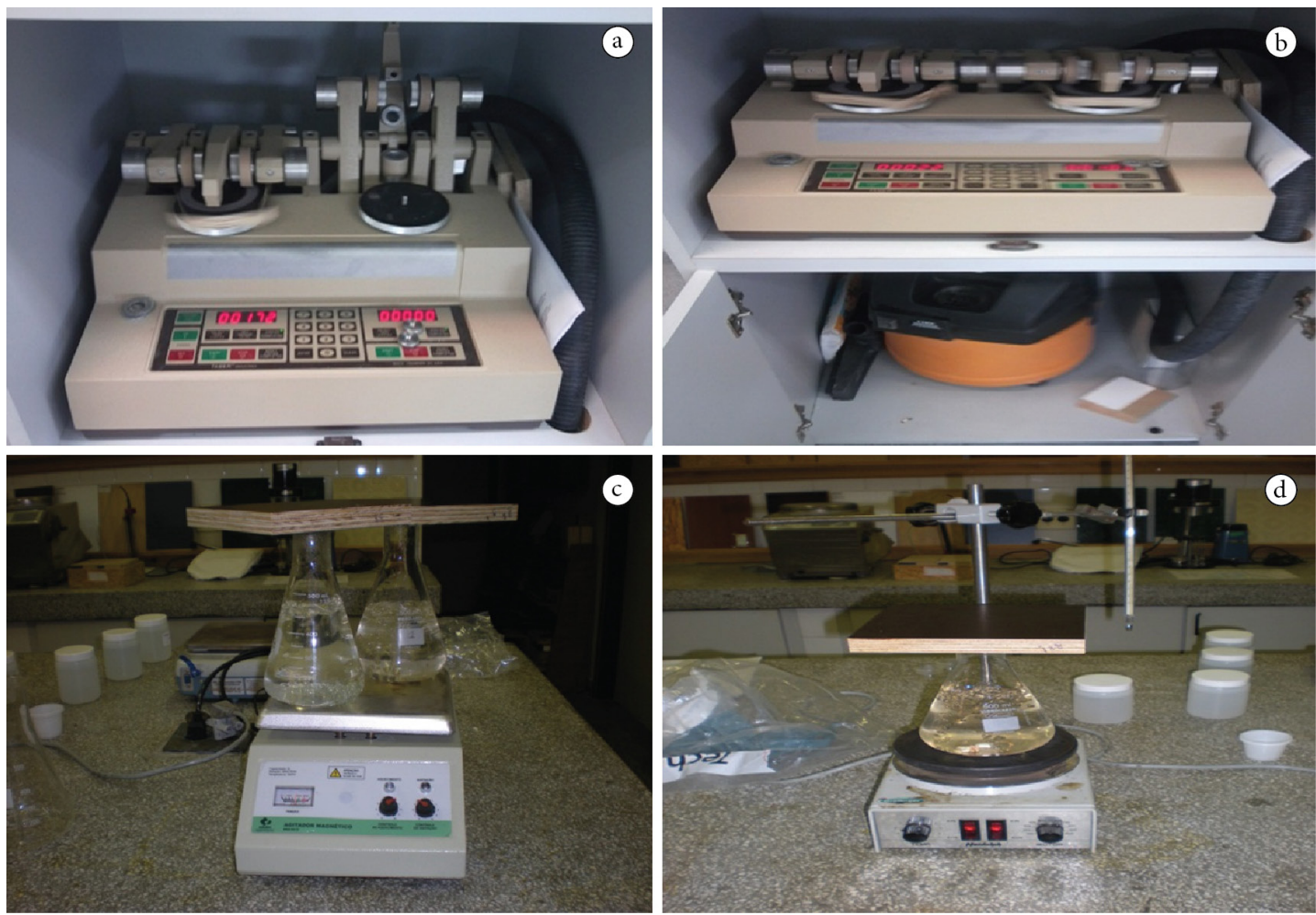

Figura 1. (A) e (B) Ensaios de abrasão; (C) e (D) Ensaios de absorção de vapor.

Figure 1. (A) and (B) Tests of abrasion. (C) and (D) Tests of vapor absorption.

Ao se analisarem as variáveis-resposta em sua escala original, procedeu-se à análise de resíduos para verificação das pressuposições de normalidade, através do teste de Shapiro-Wilk, e de homogeneidade de variâncias, através do teste de Bartlett. Todas as variâncias das amostras analisadas, associadas às variáveis de respostas e provenientes dos diferentes tratamentos, apresentavam-se homogêneas.

Foram consideradas interações de ordens 2, 3 e 4 entre os fatores. Em seguida, através da análise de correlação, foi verificado se houve correlações entre as variáveis- resposta. Todos os testes foram aplicados no nível de 95\% de significância e as análises foram realizadas no software estatístico R.

\section{RESULTADOS E DISCUSSÃO}

Os resultados de todos os ensaios estão apresentados na Tabela 2. Ao analisar a abrasão em sua escala original, no nível de significância de 5\%, os fatores Gramatura e Temperatura se mostraram significativos para os ensaios de abrasão. Uma interação de ordem 2 (Temperatura:Pressão) e três interações de ordem 3 (Gramatura:Temperatura:Tempo, Gramatura: Pressão:Tempo e Temperatura:Pressão:Tempo) mostraram-se significativas, indicando que os efeitos compreendidos em tais interações devem ser avaliados conjuntamente.

Aoseanalisaravariável 'absorção devapor' emuma hora, em sua escala original, no nível de significância de $5 \%$, nenhum fator se mostrou significativo. Uma interação de ordem 3 (temperatura:pressão:tempo) se mostrou significativa, indicando que os efeitos dos fatores compreendidos em tais interações devem ser avaliados conjuntamente.

Ao se analisar a variável 'absorção de vapor' em 2 horas, em sua escala original, no nível de significância de 5\%, apenas o fator 'pressão' se mostrou significativo. Uma interação de ordem 3 (gramatura:temperatura:tempo) se mostrou significativa, indicando que os efeitos dos fatores compreendidos em tais interações devem ser avaliados conjuntamente. 


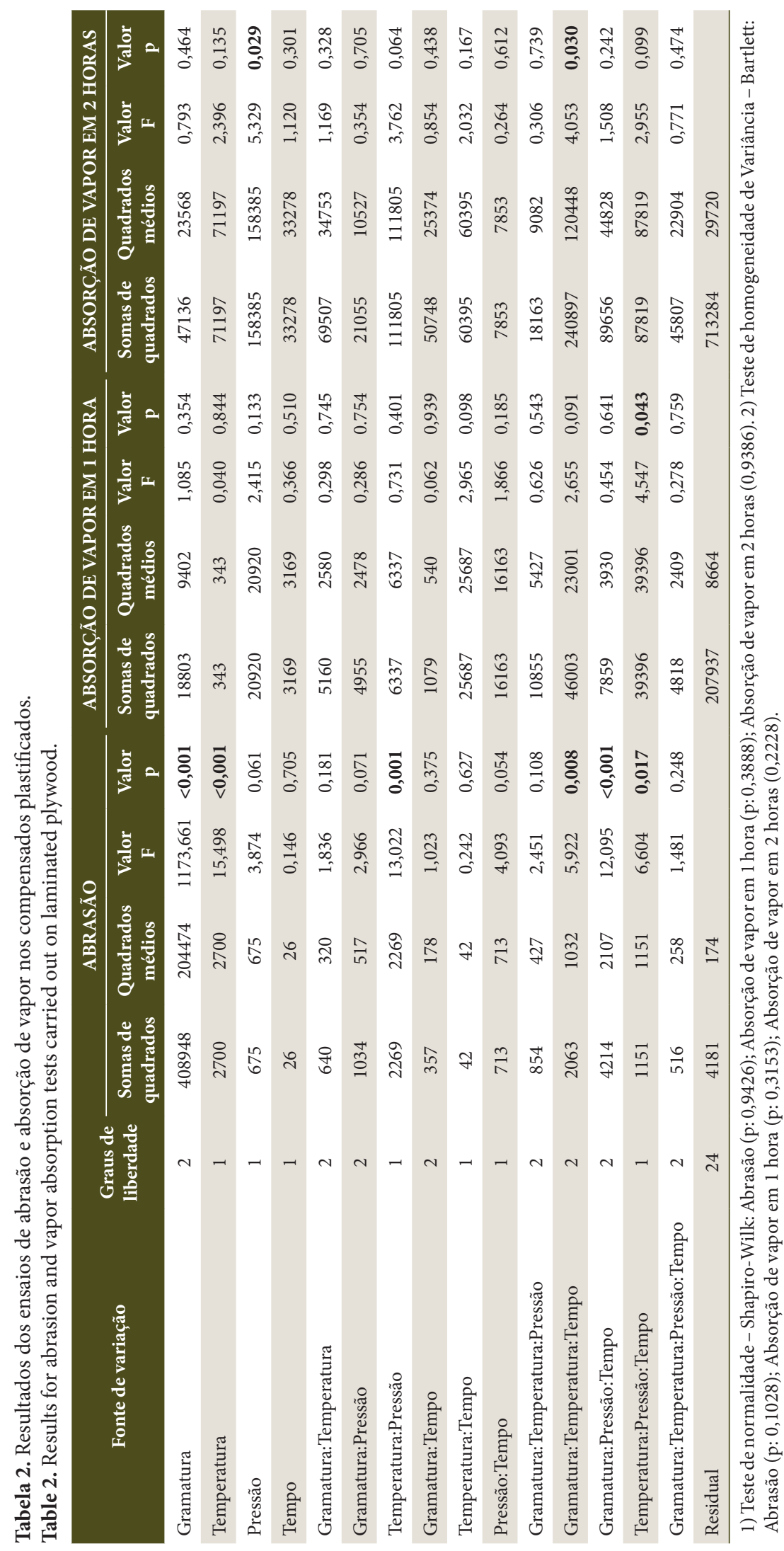




\subsection{Análise da interação temperatura:pressão para abrasão}

Os resultados das interações temperatura:pressão para abrasão estão apresentados na Tabela 3. A análise do efeito da pressão para cada temperatura é representada por letras maiúsculas e do efeito da temperatura para pressão é representada por letras minúsculas. A pressão de $18 \mathrm{kgf} / \mathrm{cm}^{2}$ apresentou resistência média à abrasão significativamente maior do que a pressão de $16 \mathrm{kgf} / \mathrm{cm}^{2}$ para a temperatura de $130{ }^{\circ} \mathrm{C}$. Para a temperatura de $140{ }^{\circ} \mathrm{C}$, não houve diferença significativa entre as pressões $16 \mathrm{e}$ $18 \mathrm{kgf} / \mathrm{cm}^{2}$. A temperatura de $140{ }^{\circ} \mathrm{C}$ apresentou resistência média à abrasão significativamente maior do que a temperatura de $130{ }^{\circ} \mathrm{C}$, para a pressão de $16 \mathrm{kgf} / \mathrm{cm}^{2}$. Para a pressão de $18 \mathrm{kgf} / \mathrm{cm}^{2}$, não houve diferença significativa entre as temperaturas de $130 \mathrm{e}$ $140{ }^{\circ} \mathrm{C}$.

Os resultados obtidos mostram que tratamentos com valores mais altos em critérios de pressão e temperatura proporcionaram melhores resultados para resistência à abrasão; contudo, estes resultados não são constantes em todos os tratamentos.

\subsection{Análise da interação gramatura:temperatura:tempo para abrasão}

Os resultados das interações gramatura: temperatura:tempo para abrasão estão apresentados

Tabela 3. Comparações múltiplas entre pressão: temperatura para ensaios de abrasão.

Table 3. Multiple comparisons from pressure: temperature for abrasion tests.

\begin{tabular}{ccc} 
Pressão & \multicolumn{2}{c}{ Temperatura $\left({ }^{\circ} \mathbf{C}\right)$} \\
\cline { 2 - 3 }$\left(\mathbf{k g f} / \mathbf{c m}^{\mathbf{2}}\right)$ & $\mathbf{1 3 0}$ & $\mathbf{1 4 0}$ \\
\hline 16 & $367,50(30,27) \mathrm{Bb}$ & $396,25(27,91) \mathrm{Aa}$ \\
18 & $388,75(26,26) \mathrm{Aa}$ & $390,00(28,86) \mathrm{Aa}$ \\
\hline
\end{tabular}

na Tabela 4. Para análise do efeito do tempo para cada temperatura e gramatura, este é representado por letras maiúsculas, e o efeito da temperatura para cada tempo e gramatura, é representado em letras minúsculas.

Para o tempo de 6 minutos e gramatura de $120 \mathrm{~g} / \mathrm{m}^{2}$, a resistência média à abrasão para a temperatura de $140{ }^{\circ} \mathrm{C}$ é significativamente maior do que para a temperatura de $130^{\circ} \mathrm{C}$. Para o tempo de 8 minutos e a gramatura de $120 \mathrm{~g} / \mathrm{m}^{2}$, não houve diferença significativa entre os tratamentos. Para a temperatura de $140^{\circ} \mathrm{C}$ e a gramatura de $120 \mathrm{~g} / \mathrm{m}^{2}$, a resistência média à abrasão para o tempo de 6 minutos é siginificativamente maior do que para o tempo de 8 minutos. Para a temperatura de $130{ }^{\circ} \mathrm{C}$ e a gramatura de $120 \mathrm{~g} / \mathrm{m}^{2}$, não houve diferença significativa entre os tratamentos.

Para o tempo de 8 minutos e gramatura de $165 \mathrm{~g} / \mathrm{m}^{2}$, a resistência média à abrasão para a temperatura de $140{ }^{\circ} \mathrm{C}$ é significativamente maior do que para a temperatura de $130{ }^{\circ} \mathrm{C}$. Para o tempo de 6 minutos e a gramatira de $165 \mathrm{~g} / \mathrm{m}^{2}$, não houve diferença significativa entre os tratamentos. Para a temperatura de $140^{\circ} \mathrm{C}$ e a gramatura de $165 \mathrm{~g} / \mathrm{m}^{2}$, a resistência média à abrasão para o tempo de 8 minutos é siginificativamente maior do que para o tempo de 6 minutos. Para a temperatura de $130{ }^{\circ} \mathrm{C}$ e a gramatura de $165 \mathrm{~g} / \mathrm{m}^{2}$, não houve diferença significativa entre os tratamentos.

Para o tempo de 8 minutos e a gramatura de $210 \mathrm{~g} / \mathrm{m}^{2}$, a resistência média à abrasão para a temperatura de $140{ }^{\circ} \mathrm{C}$ é significativamente maior do que para a temperatura de $130{ }^{\circ} \mathrm{C}$. Para o tempo de 6 minutos e a gramatura de $210 \mathrm{~g} / \mathrm{m}^{2}$, não houve diferença significativa. Para a temperatura de $130{ }^{\circ} \mathrm{C}$ com a gramatura de $210 \mathrm{~g} / \mathrm{m}^{2}$, bem como para a temperatura de $140{ }^{\circ} \mathrm{C}$ com a gramatura de

Tabela 4. Comparações múltiplas entre tempo:temperatura:gramatura para ensaios de abrasão.

Table 4. Multiple comparisons among time:temperature:grammage for abrasion tests.

\begin{tabular}{|c|c|c|c|}
\hline \multirow{2}{*}{ Gramatura $\left(\mathrm{g} / \mathrm{m}^{2}\right)$} & \multirow{2}{*}{ Tempo (min) } & \multicolumn{2}{|c|}{ Temperatura $\left({ }^{\circ} \mathrm{C}\right)$} \\
\hline & & 130 & 140 \\
\hline \multirow{2}{*}{120} & 6 & $257,50(7,50) \mathrm{Ab}$ & $293,75(8,00) \mathrm{Aa}$ \\
\hline & 8 & $266,87(14,26) \mathrm{Aa}$ & $271,87(5,53) \mathrm{Ba}$ \\
\hline \multirow{2}{*}{165} & 6 & $388,75(5,54) \mathrm{Aa}$ & $377,50(11,64) \mathrm{Ba}$ \\
\hline & 8 & $378,13(16,18) \mathrm{Ab}$ & $398,75(17,25) \mathrm{Aa}$ \\
\hline \multirow{2}{*}{210} & 6 & $488,75(8,07) \mathrm{Aa}$ & $503,13(2,77) \mathrm{Aa}$ \\
\hline & 8 & $488,75(10,08) \mathrm{Ab}$ & $513,75(6,81) \mathrm{Aa}$ \\
\hline
\end{tabular}


$210 \mathrm{~g} / \mathrm{m}^{2}$, as mudanças de tempo de prensagem para os tratamentos não tiveram diferenças significativas entre si. Os resultados mostram que tratamentos diferem entre si sem uma constância para os critérios de tempo e temperatura.

Conforme resultados apresentados na Tabela 5, a gramatura de $210 \mathrm{~g} / \mathrm{m}^{2}$ apresentou resistência média à abrasão significativamente maior do que as demais gramaturas para todas as combinações de tratamentos, assim como, neste critério, a gramatura de $165 \mathrm{~g} / \mathrm{m}^{2}$ mostrou-se maior do que a gramatura de $120 \mathrm{~g} / \mathrm{m}^{2}$ para todas as combinações de tratamentos.

\subsection{Análise da interação gramatura:pressão:tempo para abrasão}

Os resultados das interações gramatura: pressão:tempo para abrasão estão apresentados nas Tabelas 6 e 7. A análise do efeito do tempo para cada pressão e gramatura é representada por letras maiúsculas, e o efeito da pressão para cada tempo e gramatura é representado por letras minúsculas.

Para o tempo de 8 minutos e a gramatura de $120 \mathrm{~g} / \mathrm{m}^{2}$, a resistência média à abrasão para a pressão de $18 \mathrm{kgf} / \mathrm{cm}^{2}$ é significativamente maior do que para a pressão de $16 \mathrm{kgf} / \mathrm{cm}^{2}$. Para o tempo de 6 minutos e a gramatura de $120 \mathrm{~g} / \mathrm{m}^{2}$, não houve diferença significativa entre os tratamentos. Para a pressão de $16 \mathrm{kgf} / \mathrm{cm}^{2}$ com a gramatura de $120 \mathrm{~g} / \mathrm{m}^{2}$, bem como a pressão de $18 \mathrm{kgf} / \mathrm{cm}^{2}$ com a gramatura de $120 \mathrm{~g} / \mathrm{m}^{2}$, as mudanças de tempo de prensagem para os tratamentos não tiveram diferenças significativas entre si.

Para o tempo de 8 minutos e a gramatura de $165 \mathrm{~g} / \mathrm{m}^{2}$, a resistência média à abrasão para a pressão de $18 \mathrm{kgf} / \mathrm{cm}^{2}$ é significativamente maior do que para a pressão de $16 \mathrm{kgf} / \mathrm{cm}^{2}$. Para o tempo de 6 minutos e a gramatura de $165 \mathrm{~g} / \mathrm{m}^{2}$, não houve diferença significativa entre tratamentos. Para a pressão de $16 \mathrm{kgf} / \mathrm{cm}^{2}$ e a gramatura de $165 \mathrm{~g} / \mathrm{m}^{2}$,

Tabela 5. Comparações múltiplas entre tempo:temperatura:gramatura para ensaios de abrasão.

Table 5. Multiple comparisons among time:temperature:grammage for abrasion tests.

\begin{tabular}{|c|c|c|c|c|c|c|}
\hline Temperatura $\left({ }^{\circ} \mathrm{C}\right)$ & Tempo (min) & Gramatura $\left(\mathrm{g} / \mathrm{m}^{2}\right)$ & Média (dp) & & Grupo & \\
\hline \multirow{3}{*}{130} & \multirow{3}{*}{6} & 120 & $257,50(7,50)$ & & \multirow{3}{*}{$\mathrm{b}$} & \multirow[t]{3}{*}{ c } \\
\hline & & 165 & $388,75(5,54)$ & & & \\
\hline & & 210 & $488,75(8,07)$ & $\mathrm{a}$ & & \\
\hline \multirow{3}{*}{130} & \multirow{3}{*}{8} & 120 & $266,88(14,27)$ & & \multirow{3}{*}{$\mathrm{b}$} & \multirow[t]{3}{*}{ c } \\
\hline & & 165 & $378,13(16,18)$ & & & \\
\hline & & 210 & $488,75(10,08)$ & a & & \\
\hline \multirow{3}{*}{140} & \multirow{3}{*}{6} & 120 & $293,75(8,00)$ & & \multirow{3}{*}{$\mathrm{b}$} & \multirow[t]{3}{*}{ c } \\
\hline & & 165 & $377,50(11,64)$ & & & \\
\hline & & 210 & $503,125(2,77)$ & a & & \\
\hline \multirow{3}{*}{140} & \multirow{3}{*}{8} & 120 & $271,87(5,53)$ & & \multirow{3}{*}{$\mathrm{b}$} & c \\
\hline & & 165 & $398,75(17,25)$ & & & \\
\hline & & 210 & $513,75(6,81)$ & a & & \\
\hline
\end{tabular}

Tabela 6. Comparações múltiplas entre tempo:pressão:gramatura para ensaios de abrasão.

Table 6. Multiple comparisons among time:pressure:grammage for abrasion tests.

\begin{tabular}{cccc} 
Gramatura $\left(\mathbf{g} / \mathbf{m}^{2}\right)$ & Tempo $(\mathbf{m i n})$ & $\mathbf{1 6}$ & $\mathbf{1 8}$ \\
\cline { 3 - 4 } & 6 & $276,25(18,19) \mathrm{Aa}$ & $275,00(2,89) \mathrm{Aa}$ \\
\multirow{2}{*}{120} & 8 & $259,38(10,92) \mathrm{Ab}$ & $279,38(7,24) \mathrm{Aa}$ \\
\multirow{2}{*}{165} & 6 & $388,75(5,54) \mathrm{Aa}$ & $377,50(11,64) \mathrm{Ba}$ \\
& 8 & $365,00(9,79) \mathrm{Bb}$ & $411,88(12,97) \mathrm{Aa}$ \\
\multirow{2}{*}{210} & 6 & $490,00(8,72) \mathrm{Ba}$ & $501,88(2,77) \mathrm{Aa}$ \\
& 8 & $511,88(7,17) \mathrm{Aa}$ & $490,63(11,20) \mathrm{Ab}$ \\
\hline
\end{tabular}


Tabela 7. Comparações múltiplas entre tempo:pressão:gramatura para ensaios de abrasão Table 7. Multiple comparisons among time:pressure:grammage for abrasion tests.

\begin{tabular}{|c|c|c|c|c|c|c|}
\hline Pressão $\left(\mathrm{kgf} / \mathrm{cm}^{2}\right)$ & Tempo (min) & Gramatura $\left(\mathrm{g} / \mathrm{m}^{2}\right)$ & Média (dp) & & Grupo & \\
\hline \multirow{3}{*}{16} & \multirow{3}{*}{6} & 120 & $276,25(18,19)$ & & \multirow{3}{*}{$\mathrm{b}$} & c \\
\hline & & 165 & $388,75(5,54)$ & & & \\
\hline & & 210 & $490,00(8,72)$ & $\mathrm{a}$ & & \\
\hline \multirow{3}{*}{16} & \multirow{3}{*}{8} & 120 & $259,38(10,92)$ & & \multirow{3}{*}{$\mathrm{b}$} & c \\
\hline & & 165 & $365,00(9,79)$ & & & \\
\hline & & 210 & $511,88(7,17)$ & a & & \\
\hline \multirow{3}{*}{18} & \multirow{3}{*}{6} & 120 & $275,00(2,89)$ & & \multirow{3}{*}{$\mathrm{b}$} & c \\
\hline & & 165 & $377,50(11,64)$ & & & \\
\hline & & 210 & $501,88(2,77)$ & $\mathrm{a}$ & & \\
\hline \multirow{3}{*}{18} & \multirow{3}{*}{8} & 120 & $279,38(7,24)$ & & \multirow{3}{*}{$\mathrm{b}$} & c \\
\hline & & 165 & $411,88(12,97)$ & & & \\
\hline & & 210 & $490,63(11,20)$ & a & & \\
\hline
\end{tabular}

a resistência média à abrasão para o tempo de 6 minutos é significativamente maior do que para o tempo de 8 minutos. Ao contrário, para a pressão de $18 \mathrm{kgf} / \mathrm{cm}^{2}$, a resistência média à abrasão para o tempo de 8 minutos é significativamente maior do que para o tempo de 6 minutos.

Para o tempo de 8 minutos e a gramatura de $210 \mathrm{~g} / \mathrm{m}^{2}$, a resistência média à abrasão para a pressão de $16 \mathrm{kgf} / \mathrm{cm}^{2}$ é significativamente maior do que para a pressão de $18 \mathrm{kgf} / \mathrm{cm}^{2}$. Para o tempo de 6 minutos e a gramatura de $210 \mathrm{~g} / \mathrm{m}^{2}$, não houve diferença significativa entre os tratamentos. Para a pressão de $16 \mathrm{kgf} / \mathrm{cm}^{2}$ e a gramatura de $210 \mathrm{~g} / \mathrm{m}^{2}$, a resistência média à abrasão para o tempo de 8 minutos é significativamente maior do que para o tempo de 6 minutos. Ao contrário, para a pressão de $18 \mathrm{kgf} / \mathrm{cm}^{2}$, a resistência média à abrasão para o tempo de 6 minutos é significativamente maior do que para o tempo de 8 minutos. Os resultados mostram que os tratamentos diferem entre si sem uma constância de valores para os critérios de tempo e pressão.

A gramatura de $210 \mathrm{~g} / \mathrm{m}^{2}$ apresentou resistência média à abrasão significativamente maior do que as demais gramaturas para todas as combinações de tratamentos (pressão e tempo). A gramatura de $165 \mathrm{~g} / \mathrm{m}^{2}$ apresentou resistência média à abrasão significativamente maior do que a gramatura de $120 \mathrm{~g} / \mathrm{m}^{2}$ para todas as combinações de tratamentos (pressão e tempo).

\subsection{Análise da interação temperatura:pressão:tempo para abrasão}

Os resultados das interações temperatura: pressão:tempo para abrasão estão apresentados na Tabela 8. A análise do efeito do tempo para cada temperatura e pressão é representada por letras maiúsculas, e o efeito da pressão para cada tempo e temperatura é representado em letras minúsculas.

Para o tempo de 6 minutos e a temperatura de $130{ }^{\circ} \mathrm{C}$, a resistência média à abrasão para a pressão de $18 \mathrm{kgf} / \mathrm{cm}^{2}$ foi significativamente maior do que para a pressão de $16 \mathrm{kgf} / \mathrm{cm}^{2}$. Para o tempo de 8 minutos e a temperatura de $130{ }^{\circ} \mathrm{C}$, a resistência média à abrasão para a pressão de $18 \mathrm{kgf} / \mathrm{cm}^{2}$ também foi significativamente maior do que para a pressão de $16 \mathrm{kgf} / \mathrm{cm}^{2}$.

Para a pressão de $16 \mathrm{kgf} / \mathrm{cm}^{2} \mathrm{com}$ a temperatura de $130^{\circ} \mathrm{C}$, bem como a pressão de $18 \mathrm{kgf} / \mathrm{cm}^{2}$ com a temperatura de $130^{\circ} \mathrm{C}$, as mudanças de tempo de prensagem para os tratamentos não apresentaram diferenças significativas entre si. Para o tempo de 6 minutos e a temperatura de $140{ }^{\circ} \mathrm{C}$, a resistência média à abrasão para a pressão de $16 \mathrm{kgf} / \mathrm{cm}^{2}$ é significativamente maior do que para a pressão de $18 \mathrm{kgf} / \mathrm{cm}^{2}$. Para o tempo de 8 minutos e a temperatura de $140^{\circ} \mathrm{C}$, não houve diferença significativa entre os tratamentos.

Para a pressão de $18 \mathrm{kgf} / \mathrm{cm}^{2}$ e a temperatura de $140{ }^{\circ} \mathrm{C}$, a resistência média à abrasão para o tempo 
de 8 minutos é significativamente maior do que para o tempo de 6 minutos. Para a pressão de $16 \mathrm{kgf} / \mathrm{cm}^{2}$ e a temperatura de $140{ }^{\circ} \mathrm{C}$, não houve diferença significativa entre os tratamentos.

Conforme apresentado na Tabela 9, a temperatura de $140^{\circ} \mathrm{C}$ apresentou resistência à abrasão média significativamente maior do que a temperatura de $130^{\circ} \mathrm{C}$ para as combinações de tratamentos com pressão de $16 \mathrm{kgf} / \mathrm{cm}^{2}$ e ambos os tempos. Para as combinações de tratamento com pressão de $18 \mathrm{kgf} / \mathrm{cm}^{2}$ e ambos os tempos, não houve diferença significativa entre as temperaturas de 130 e $140^{\circ} \mathrm{C}$.

\subsection{Análise da interação}

temperatura:pressão:tempo para absorção de vapor em uma hora

Os resultados das interações temperatura: pressão:tempo para absorção de vapor em uma hora estão apresentados nas Tabelas 10 e 11 . A análise do efeito do tempo para cada temperatura e pressão é representada por letras maiúsculas, e o efeito da pressão para cada tempo e temperatura é representado em letras minúsculas.

Para o tempo de 6 minutos e a temperatura de $130{ }^{\circ} \mathrm{C}$, a absorção de vapor em uma hora para a pressão de $16 \mathrm{kgf} / \mathrm{cm}^{2}$ é significativamente menor do que para a pressão de $18 \mathrm{kgf} / \mathrm{cm}^{2}$. Para o tempo de 8 minutos e a temperatura de $130^{\circ} \mathrm{C}$, não houve diferença significativa entre os tratamentos.

Para a pressão de $16 \mathrm{kgf} / \mathrm{cm}^{2}$ e a temperatura de $130{ }^{\circ} \mathrm{C}$, a absorção de vapor em uma hora para o tempo de prensagem de 6 minutos é significativamente menor do que para o tempo de prensagem de 8 minutos. Para a pressão de $18 \mathrm{kgf} / \mathrm{cm}^{2}$ e a temperatura de $130{ }^{\circ} \mathrm{C}$, não houve diferença significativa entre os tratamentos. Para

Tabela 8. Comparações múltiplas entre tempo:pressão:temperatura para ensaios de abrasão.

Table 8. Multiple comparisons among time:pressure:temperature for abrasion tests.

\begin{tabular}{cccc} 
Temperatura $\left({ }^{\circ} \mathbf{C}\right)$ & Tempo (min) & $\mathbf{1 6}$ & Pressão $\left(\mathbf{k g f} / \mathbf{c m}^{2}\right)$ \\
\cline { 3 - 4 } & 6 & $366,67(42,25) \mathrm{Ab}$ & $390,00(42,52) \mathrm{Aa}$ \\
\multirow{2}{*}{130} & 8 & $368,33(47,41) \mathrm{Ab}$ & $387,50(34,99) \mathrm{Aa}$ \\
\multirow{2}{*}{140} & 6 & $403,33(36,12) \mathrm{Aa}$ & $379,58(41,03) \mathrm{Bb}$ \\
& 8 & $389,17(45,87) \mathrm{Aa}$ & $400,42(44,01) \mathrm{Aa}$ \\
\hline
\end{tabular}

Tabela 9. Comparações múltiplas entre tempo:pressão:temperatura para ensaios de abrasão. Table 9. Multiple comparisons among time:pressure:temperature for abrasion tests.

\begin{tabular}{|c|c|c|c|c|c|}
\hline Pressão $\left(\mathrm{kgf} / \mathrm{cm}^{2}\right)$ & Tempo (min) & Temperatura $\left(\mathrm{kgf} / \mathrm{cm}^{2}\right)$ & Média (dp) & \multicolumn{2}{|c|}{ Grupo } \\
\hline \multirow{2}{*}{16} & \multirow{2}{*}{6} & 130 & $366,67(42,25)$ & & \multirow[t]{2}{*}{$b$} \\
\hline & & 140 & $403,33(36,12)$ & $\mathrm{a}$ & \\
\hline \multirow{2}{*}{16} & \multirow{2}{*}{8} & 130 & $368,33(47,41)$ & & \multirow[t]{2}{*}{$\mathrm{b}$} \\
\hline & & 140 & $389,17(45,87)$ & $\mathrm{a}$ & \\
\hline \multirow{2}{*}{18} & \multirow{2}{*}{6} & 130 & $390,00(42,52)$ & a & \\
\hline & & 140 & $379,58(41,03)$ & a & \\
\hline \multirow{2}{*}{18} & \multirow{2}{*}{8} & 130 & $387,50(34,99)$ & a & \\
\hline & & 140 & $400,42(44,01)$ & a & \\
\hline
\end{tabular}

Tabela 10. Comparações múltiplas entre tempo:pressão:temperatura.

Table 10. Multiple comparisons among time:pressure:temperature.

\begin{tabular}{cccc} 
Temperatura $\left({ }^{\circ} \mathbf{C}\right)$ & Tempo (min) & $\mathbf{1 6}$ & Pressão $\left(\mathbf{k g f} / \mathbf{c m}^{\mathbf{2}}\right)$ \\
\cline { 3 - 4 } & 6 & $63,64(13,93) \mathrm{Ab}$ & $176,40(60,06) \mathrm{Aa}$ \\
\multirow{2}{*}{130} & 8 & $127,62(44,70) \mathrm{Aa}$ & $52,39(10,61) \mathrm{Ba}$ \\
\multirow{2}{*}{140} & 6 & $57,03(11,76) \mathrm{Aa}$ & $101,17(30,39) \mathrm{Aa}$ \\
& 8 & $98,96(39,55) \mathrm{Aa}$ & $184,29(41,36) \mathrm{Aa}$ \\
\hline
\end{tabular}


Tabela 11. Comparações múltiplas entre tempo:pressão:temperatura.

Table 11. Multiple comparisons among time:pressure:temperature.

\begin{tabular}{|c|c|c|c|c|c|}
\hline Pressão $\left(\mathrm{kgf} / \mathrm{cm}^{2}\right)$ & Tempo (min) & Temperatura $\left(\mathrm{kgf} / \mathrm{cm}^{2}\right)$ & Média (dp) & \multicolumn{2}{|c|}{ Grupo } \\
\hline \multirow{2}{*}{16} & \multirow{2}{*}{6} & 130 & $63,64(13,93)$ & $\mathrm{a}$ & \\
\hline & & 140 & $57,03(11,75)$ & a & \\
\hline \multirow{2}{*}{16} & \multirow{2}{*}{8} & 130 & $127,62(44,70)$ & $\mathrm{a}$ & \\
\hline & & 140 & $98,96(39,54)$ & $\mathrm{a}$ & \\
\hline \multirow{2}{*}{18} & \multirow{2}{*}{6} & 130 & $176,41(60,06)$ & $\mathrm{a}$ & \\
\hline & & 140 & $101,17(30,39)$ & a & \\
\hline \multirow{2}{*}{18} & \multirow{2}{*}{8} & 130 & $52,39(10,62)$ & & b \\
\hline & & 140 & $184,29(41,35)$ & a & \\
\hline
\end{tabular}

Tabela 12. Comparações entre as médias para a variável pressão.

Table 12. Comparisons between the averages for the variable pressure.

\begin{tabular}{ccc} 
Pressão & Média(dp) & \multicolumn{2}{c}{ Grupo } \\
\hline 16 & $187,42(26,07)$ & \\
18 & $302,31(48,41)$ & a \\
\hline
\end{tabular}

a temperatura de $140{ }^{\circ} \mathrm{C}$, as interações entre as variáveis não foram significativas entre si.

A temperatura de $140{ }^{\circ} \mathrm{C}$ apresentou absorção média de vapor em uma hora significativamente maior do que a temperatura de $130{ }^{\circ} \mathrm{C}$ para a combinação do tratamento com pressão de $18 \mathrm{kgf} / \mathrm{cm}^{2}$ e tempo de 8 minutos. Para os demais tratamentos, não houve diferença significativa entre as temperaturas de 130 e $140{ }^{\circ} \mathrm{C}$.

\subsection{Análise do efeito da pressão para a absorção de vapor em duas horas}

A Tabela 12 apresenta a análise do efeito da pressão para a variável 'absorção de vapor’ em duas horas, pois o fator não se encontra na interação que foi significativa.

Para a absorção de vapor em duas horas, a pressão de $18 \mathrm{kgf} / \mathrm{cm}^{2}$ apresentou absorção de vapor média significativamente maior do que a pressão de $16 \mathrm{kgf} / \mathrm{cm}^{2}$.

3.7. Análise da interação gramatura: temperatura: tempo para absorção de vapor em duas horas

Os resultados das interações gramatura: temperatura:tempo para absorção de vapor em duas horas estão apresentados nas Tabelas 13 e 14. A análise do efeito do tempo para cada temperatura e gramatura é representada por letras maiúsculas, e o efeito da temperatura para cada tempo e gramatura é representado em letras minúsculas.

Para a gramatura de $120 \mathrm{~g} / \mathrm{m}^{2}$, as interações entre tempo e temperatura não foram significativas entre si. Para o tempo de 8 minutos e a gramatura de $165 \mathrm{~g} / \mathrm{m}^{2}$, a absorção de vapor em duas horas para a temperatura de $130^{\circ} \mathrm{C}$ é significativamente menor do que para a temperatura de $140{ }^{\circ} \mathrm{C}$.

Para o tempo de 6 minutos e a gramatura de $165 \mathrm{~g} / \mathrm{m}^{2}$, não houve diferença significativa. Para a temperatura de $130^{\circ} \mathrm{C}$ e a gramatura de $165 \mathrm{~g} / \mathrm{m}^{2}$, a absorção de vapor em duas horas para o tempo de 8 minutos é siginificativamente menor do que para o tempo de 6 minutos. Para a temperatura de $140{ }^{\circ} \mathrm{C}$ e a gramatura de $165 \mathrm{~g} / \mathrm{m}^{2}$, não houve diferença significativa. Para a gramatura de $210 \mathrm{~g} / \mathrm{m}^{2}$, as interações também não foram significativas.

\section{CONCLUSÕES}

Os ensaios de abrasão indicaram que o produto revestido com filme fenólico de maior gramatura é mais resistente e não tem influência significativa dos parâmetros de prensagem do papel fenólico.

A absorção de umidade através da aplicação de vapor comprovou que os parâmetros de prensagem e a gramatura do filme fenólico não interferem de forma constante e significativa na absorção de vapor pelo compensado plastificado.

O número de reutilizações como fôrmas de concreto seriam proporcionais aos valores de 
Tabela 13. Comparações múltiplas entre tempo:temperatura:gramatura.

Table 13. Multiple comparisons among time:temperature:grammage.

\begin{tabular}{cccc} 
Gramatura $\left(\mathbf{g} / \mathbf{m}^{2}\right)$ & Tempo $(\mathbf{m i n})$ & $\mathbf{1 3 0}$ & Temperatura $\left({ }^{\circ} \mathbf{C}\right)$ \\
\cline { 3 - 4 } & 6 & $258,53(117,52) \mathrm{Aa}$ & $226,11(85,04) \mathrm{Aa}$ \\
\multirow{2}{*}{120} & 8 & $151,49(41,20) \mathrm{Aa}$ & $367,90(116,93) \mathrm{Aa}$ \\
\multirow{2}{*}{165} & 6 & $232,67(97,98) \mathrm{Aa}$ & $183,08(47,05) \mathrm{Ba}$ \\
& 8 & $165,08(38,21) \mathrm{Ab}$ & $538,38(191,54) \mathrm{Aa}$ \\
\hline \multirow{2}{*}{210} & 6 & $155,28(58,12) \mathrm{Aa}$ & $255,54(83,49) \mathrm{Aa}$ \\
& 8 & $275,05(68,03) \mathrm{Aa}$ & $129,26(56,00) \mathrm{Aa}$ \\
\hline
\end{tabular}

Tabela 14. Comparações múltiplas entre tempo:temperatura:gramatura.

Table 14. Multiple comparisons among time:temperature:grammage.

\begin{tabular}{|c|c|c|c|c|c|}
\hline Temperatura $\left({ }^{\circ} \mathrm{C}\right)$ & Tempo (min) & Gramatura $\left(g / \mathrm{m}^{2}\right)$ & Média (dp) & & Irupo \\
\hline \multirow{3}{*}{130} & \multirow{3}{*}{6} & 120 & $258,53(117,52)$ & a & \\
\hline & & 165 & $232,67(97,98)$ & a & \\
\hline & & 210 & $155,28(58,12)$ & a & \\
\hline \multirow{3}{*}{130} & \multirow{3}{*}{8} & 120 & $151,50(41,20)$ & a & \\
\hline & & 165 & $165,08(38,21)$ & a & \\
\hline & & 210 & $275,05(68,03)$ & $\mathrm{a}$ & \\
\hline \multirow{3}{*}{140} & \multirow{3}{*}{6} & 120 & $226,11(85,04)$ & a & \\
\hline & & 165 & $183,08(47,05)$ & a & \\
\hline & & 210 & $255,54(83,49)$ & a & \\
\hline \multirow{3}{*}{140} & \multirow{3}{*}{8} & 120 & $367,91(116,93)$ & a & $\mathrm{b}$ \\
\hline & & 165 & $538,38(191,54)$ & a & \\
\hline & & 210 & $129,26(56,00)$ & & $\mathrm{b}$ \\
\hline
\end{tabular}

gramatura dos filmes fenólicos utilizados como revestimento, uma vez que a resistência à abrasão, provocada pelo lançamento de concreto nas fôrmas, está associada ao maior valor de gramatura do filme fenólico.

As opções por menores valores dos parâmetros de prensagem possibilitam ganhos econômicos na produção, tendo em vista que os mesmos não interferem nos resultados de abrasão e absorção de vapor. Portanto, recomenda-se a prensagem do filme fenólico para produção de compensado plastificado nas condições a seguir: gramatura do filme fenólico de $210 \mathrm{~g} / \mathrm{m}^{2}$, temperatura de $130{ }^{\circ} \mathrm{C}$, pressão específica de $16 \mathrm{kgf} / \mathrm{cm}^{2}$ e tempo de prensagem de 6 minutos.

\section{STATUS DA SUBMISSÃO}

Recebido: 05/06/2013

Aceito: 09/10/2013

Publicado: 31/12/2013

\section{AUTOR(ES) PARA CORRESPONDÊNCIA}

\section{Felipe Gustavo Sanches}

Departamento de Engenharia e Tecnologia

Florestal, Universidade Federal do

Paraná - UFPR, CEP 80210-170, Curitiba, PR,

Brasil

e-mail: felipe_gust@hotmail.com 


\section{REFERENNCIAS}

Araujo CRS. Emprego de fôrmas de madeira em estrutura de concreto [monografia]. Salvador: Universidade Católica do Salvador; 2010.

Associação Brasileira da Indústria de Madeira Processada Mecanicamente. Artigo Técnico: Compensado Plastificado. Curitiba: ABIMCI; 2011.

Coveright Surfaces. Coveright ${ }^{\oplus}$ - Phenolic Surface Films - Technical data and services. 04/07 E EUR 31. 2011.

Costa Junior TF. Emprego de fôrmas de madeira em estrutura de concreto [monografia]. Salvador: Escola de Engenharia, Universidade Católica de Salvador; 2008.
Iwakiri S, Olandoski DP, Leonhardt G, Brand MA. Produção de chapas de madeira compensada de cinco espécies de pinus tropicais. Ciência Florestal 2004; 11(2): 71-77.

Polzl PFK. Análise da produtividade em fábricas de painéis compensados plastificados [dissertação]. Ponta Grossa: Universidade Tecnológica Federal do Paraná; 2011.

Silva TAPRM. Optimização da cura de resinas fenólicas com a tecnologia de Infravermelho [tese]. Aveiro: Universidade de Aveiro; 2008.

Stamato GC. Resistência ao embutimento da madeira compensada [dissertação]. São Carlos: Universidade de São Paulo; 1998. 
